\title{
Modeling of Diffusion in Closed Cell Polymeric Foams
}

\author{
SACIDE ALSOY* \\ Center for the Study of Polymer-Solvent Systems \\ Department of Chemical Engineering \\ The Pennsylvania State University \\ University Park, PA 16802
}

\section{INTRODUCTION}

\begin{abstract}
Tow density polymeric foams have many applications in modern sociLety. For example, they are used in the construction, refrigeration, appliance and transportation industries when highly efficient thermal insulation materials are needed. The insulation efficiency of these materials comes from the entrapment of a low thermal conductivity blowing agent (usually a fluorocarbon) in the closed cell structure. The thermal conductivity of the entrapped gas or vapor in the cell represents an important contribution to overall foam thermal conductivity since approximately $50 \%$ of the heat transfer through the foam occurs by conduction through the gas phase. One of the major contemporary problems in foam technology deals with the decrease in their insulation value over time. The other leading problem is related to the dimensional instability of the foam. During the service life of foams, air diffuses into the cells, and a high molecular weight blowing agent diffuses out, which modifies the cell gas composition and ultimately causes a gradual loss in thermal resistivity of the foam. Cell gas pressure is also an important factor that influences the strength of foams. Since CFC production is being phased out under recent international agreement, the development of new products with alternative blowing agents requires understanding how different variables such as formulation, processing conditions, structure and geometry affect the aging behavior and dimensional
\end{abstract}

*Current address: Izmir Institute of Technology, Gaziosmanpasa Bulvari, No. 16, 35230, Cankaya-Izmir, Turkey.

JOURNAL OF CELLULAR PLASTICS Volume 35-May 1999 
stability of foams. To predict both factors, knowledge about the diffusion phenomena in the foam becomes crucial, since the mass transfer of oxygen and nitrogen in the air control the cell gas composition of the foam during short to intermediate times, and the mass transfer of blowing agent controls long term composition changes in the foam.

Several models exist in the literature to describe diffusion in foams. From a comprehensive review of the literature on this subject, models for diffusion in closed cell foams can be divided into two categories.

1. Continuous diffusion models which treat the foam as a homogeneous medium that can be characterized by an effective diffusion coefficient (Norton, 1967; Cuddihy, 1976; Ostrogorsky et al., 1986; Bomberg, 1988; Jarfelt, 1994; Brodt et al., 1995). The composition of each species in the foam is found from the analytical solution of the following equation:

$$
\frac{\partial P_{i}}{\partial t}=D_{e f f} \nabla^{2} P_{i}
$$

where $P_{i}$ is the partial pressure of component $i$ and $D_{\text {eff }}$ is the effective diffusivity of foam.

2. Discrete diffusion models which use an electrical RC network analogy to describe diffusion through a cellular structure (Cuddihy, 1967; Lee, 1973; Norton, 1982; Ostrogorsky et al., 1984; Reitz, 1984; Ostrogorsky and Glicksman, 1988a, 1988b; Bart and Du Cauze De Nazelle, 1993).

In discrete models, the diffusion process is described by the bulk polymer permeability values, and polymeric network characteristics and the effective diffusion coefficients are predicted from the measured polymer permeability combined with foam geometric parameters. This is a major advantage of discrete models over continuous ones, since direct measurements of effective foam diffusivities are lengthy and may be inaccurate as a result of cracks or holes in the cell walls. Also, continuous type models give only minimal physical insight into foam aging behavior. However, with a discrete model it is possible to evaluate the effect of different components such as foam structure, (e.g., cell wall thickness, cell size, arrangement, elongation, etc.) properties of polymer material constituting cell walls, and the morphology of the cell structure.

A discrete model developed by Bart and Du Cauze De Nazelle (1993) is the most complete model available in the literature to predict the effective diffusivity of a foam since all other discrete models referred to above neglect the solubility of the diffusant in the polymer matrix. The model was derived based on one dimensional diffusion through a three dimensional array of cubic cells consisting of walls of uniform thickness, and an effective diffusivity, $D_{\text {eff }}$, is given by 


$$
D_{e f f}=\frac{D_{p} l / d}{\left(f_{g} S_{g} / S_{p}\right)+\left(1-f_{g}\right)}
$$

where $D_{p}$ is the diffusivity in the polymer, $l$ is the thickness of the cell, $d$ is the thickness of the cell wall, $f_{g}$ is the volume fraction of the gas phase, and $S_{g}$ and $S_{p}$ are the solubilities in the gas and polymer phases, respectively. Bart and Du Cauze De Nazelle model and other discrete models available in the literature are not completely realistic in describing the diffusion through foams. First of all, the basic assumption of a steady state in the cell walls of the foam is not necessarily valid. In these models, all layers are treated the same, i.e., the boundary conditions between the cell wall and the gas phase are not considered. The relation between the gas concentration in the polymer material and the pressure in the voids is assumed to be proportional and given by Henry's law. Also, the concentration dependency of diffusion coefficient of diffusant in the polymer is neglected. Unfortunately, it is not possible to derive an explicit relationship for effective diffusivity of foams if different equilibrium relationships or concentration dependent diffusion coefficients are included. To overcome these disadvantages, an unsteady-state diffusion model is proposed, which can give a better description of diffusion through different layers.

In the first part of this work, effective diffusivities predicted from Equation (2) will be compared with experimental data. In the second part, equations for unsteady-state diffusion model will be derived and solved numerically using finite difference approximation. The development of a numerical solution procedure for solving the large number of equations in a reasonable computer time is one of the main contributions of this study. The predictions from both unsteady state model and the Bart and Du Cauze De Nazelle model will be compared with one set of available data for the uptake rate of $\mathrm{PS} / \mathrm{N}_{2}$ system. Also, the effect of some relevant parameters on both the aging profile and the dimensional stability of the foam will be discussed based on results from the simulation.

\section{COMPARISON OF PREDICTED AND EXPERIMENTAL EFFECTIVE DIFFUSIVITIES}

Effective diffusivities obtained from different sources were compared with the predictions from the model of Bart and Du Cauze De Nazelle (1993) given by Equation (2). The results are shown in Table 1. To predict the effective diffusivities from the model requires diffusivity data, in addition to information on solubility and the ratio of cell to cell wall thick- 


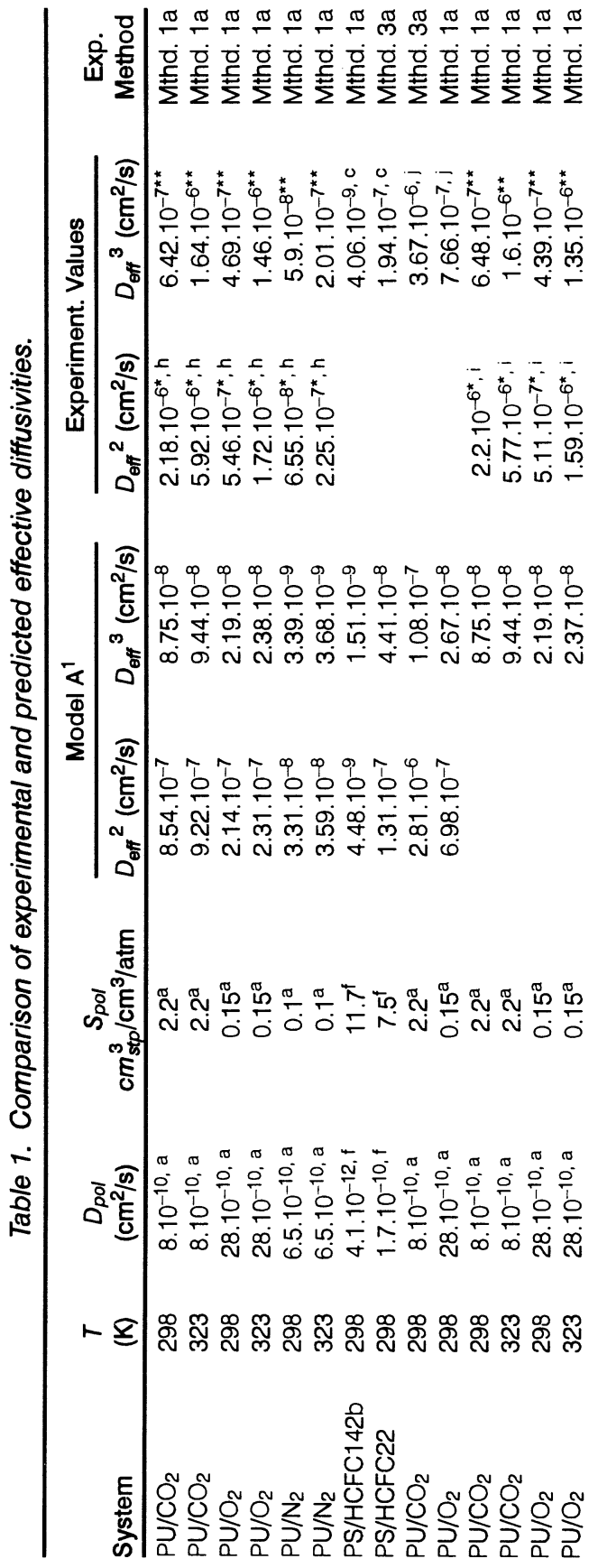




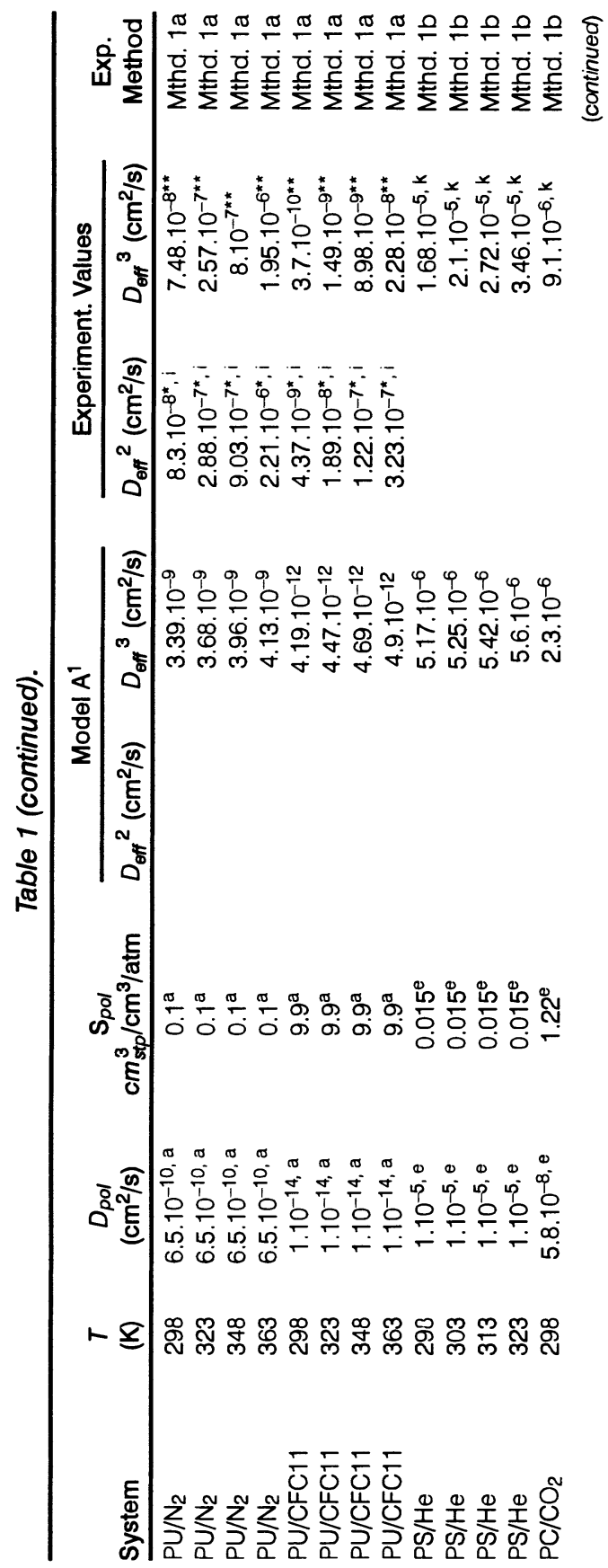




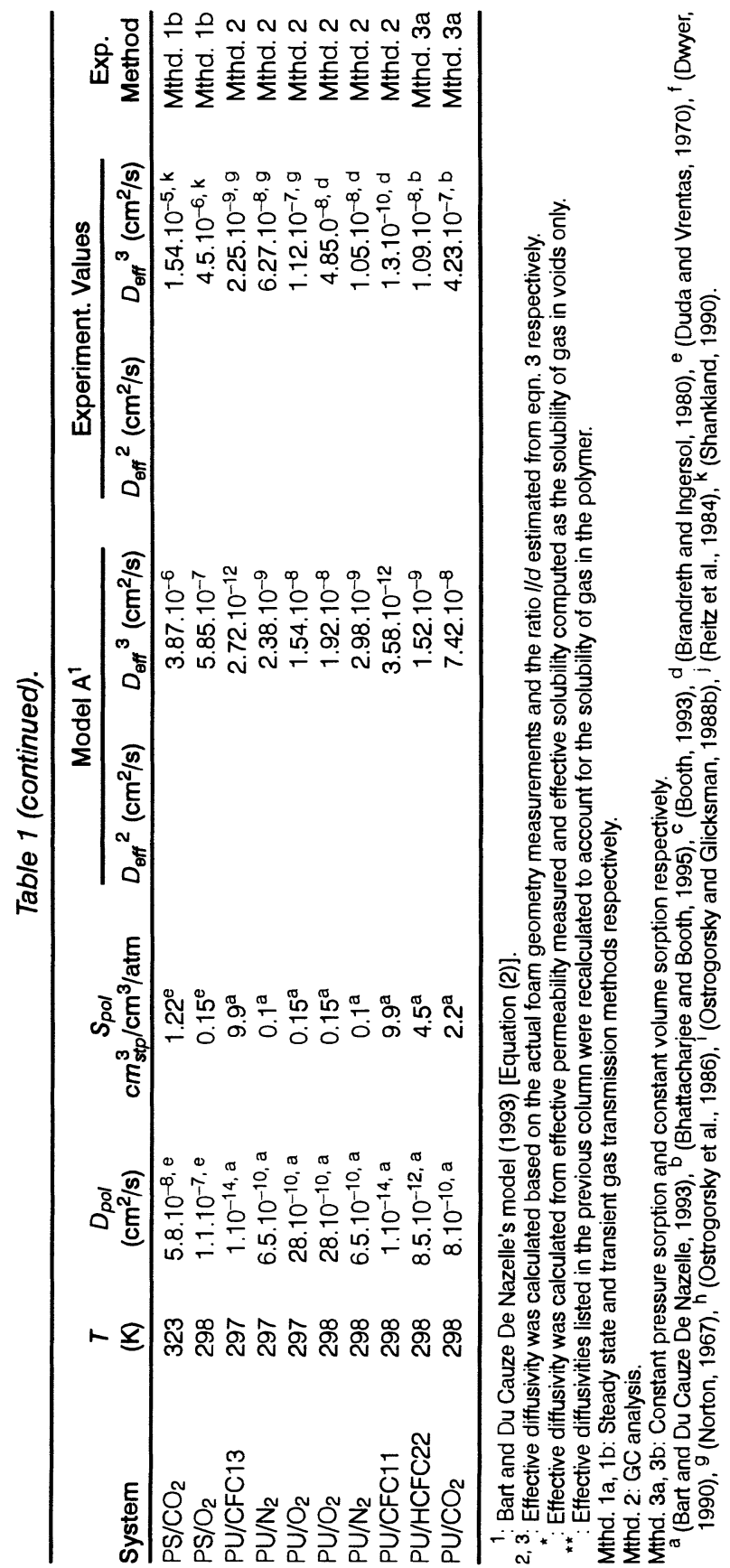


ness. Whenever actual foam geometry measurements were not available, the ratio $l / d$ was estimated from the foam density data as follows:

$$
\frac{l}{d}=\frac{1}{1-f_{g}}
$$

Volume fraction of the gas phase, $f_{g}$, is defined as follows:

$$
f_{g}=\frac{\rho_{p}-\rho_{f}}{\rho_{p}-\rho_{g}}
$$

where $\rho_{p}$ and $\rho_{f}$ are the densities of polymer and foam, respectively, and $\rho_{g}$ is the density of the cell gas. If the diffusivity is predicted with the ratio $l / d$ calculated from Equation (3), the values are one to three orders of magnitude lower than the experimental values. This large discrepancy can be partly explained by the presence of struts which are rod shaped structures located at the intersection of cell walls. Estimation of the ratio of the cell to cell wall thickness from Equation (3) assumes that all of the polymer material in the foam is present in the cell walls. This assumption would therefore result in thicker cell walls and lower predicted effective diffusivities than the experimental measurements. In actual manufactured foams, polymer is distributed between the cell walls and edges and corners of the cell, so that the distribution depends on the type of the polymer, blowing agents and processing conditions. The analysis of Reitz et al. (1984) indicates that $20 \%$ or less of the polymer material is contained in the PU foam cell walls, while Norton (1982) has microscopically determined that $50 \%$ of the total polymer is in the extruded PS foam cell walls. If the actual foam geometry measurements are used in the calculations, the model still underpredicts the experimental diffusivities for all the systems.

These results suggest that the presence of the struts cannot be the only reason for the lower predicted diffusivities. Another possible explanation for the deviation from the measured values is the existence of open cells in the cell walls. Recall that the model was derived based on the assumption that no holes exist in the cell walls and that the permeability can be calculated from the product of the diffusivity and solubility. However, in the case of cells with holes, this relationship does not correctly describe the transport of the gas into the cell interior. Depending on the size of the hole, the transport can vary from that for no hole (zero hole diameter) to that for a large hole where the permeability mechanism is negligible compared to the bulk transport by diffusion through a stagnant gas film. This would result in an increase in the effective diffusivity with increasing open cell content as shown by the data of Brandreth (1989). It should also 
be mentioned that the values for the solubility and diffusivity of the gas in the polymer phase affect the predicted values. When closed cell foams are produced by expansion, normally at high rates of strain, polymer molecules stay oriented rather than organizing randomly in the polymer phase. This orientation influences the transport and equilibrium properties in the polymer, as observed by Park (1993) and Vieth et al. (1966). Even if all of these factors which might contribute to the poor predictive ability of the model are taken into account, it is still not completely realistic in describing diffusion through foams. Therefore, a new unsteadystate model is proposed in the next section which can be extended to include the influence of concentration dependent diffusivity and nonlinear isotherms.

\section{UNSTEADY-STATE DIFFUSION MODEL}

The discrete type model developed by Bart and Du Cauze De Nazelle does not give a realistic description of diffusion in foams. The explicit relationship for the effective diffusivity given by Equation (2) was derived based on three main assumptions. The first one, which assumes that a steady-state has been reached in the polymer membrane, is not realistic, since the partitioning of the blowing agent between the gas phase and polymer phase is a slow process. It was shown that a steady-state is reached after an extended period of time which can range from months to a year (Daems et al., 1994). The second assumption uses the linear equilibrium relationship given by Henry's law, which is a relatively poor approximation to the actual sorption process in foams. Most of the polymers used in foam production are in the glassy state at room temperature. It has been observed that for glassy polymers, the sorption isotherm is nonlinear, and the sorption process is not sufficiently described by Henry's law even at low penetrant concentrations (Koros et al., 1976; Koros and Paul, 1978; Toi and Paul, 1982; Fleming and Koros, 1986). Also, ignoring the concentration dependency of the diffusion coefficient of the blowing agent in the polymer phase seems somewhat questionable. Park (1993) has shown that the diffusion coefficients of blowing agents are not constant, but rather depend on the pressure or blowing agent concentration. In typical systems the diffusivity of the blowing agent in the polymer increases with blowing agent concentration, and such concentration dependency can cause very significant deviations from the idealized constant diffusivity case (Crank, 1975) during desorption process.

To relax these assumptions, an unsteady-state diffusion model has been developed, which can be easily extended to incorporate concentration dependent diffusivity, nonlinear sorption isotherm as well as un- 
steady state behavior. Figure 1 shows the schematic of the structure of the polymer foam used to derive the equations. The model assumes that cell sizes are uniform and constant, that the foam is homogeneous and isotropic, and that no pores or holes exist in the cell walls. The thickness of the foam is much smaller than the length and width of the foam so that diffusion is only in one direction. A constant diffusivity in the polymer phase and a linear equilibrium relationship between the gas and polymer phases are assumed for illustrative purposes. However, these assumptions can be easily relaxed if related information concerning the equilibrium isotherm and the influence of concentration on the diffusivity is available. Diffusion of each species is not influenced by the presence of other components, and it is governed by Fick's law. Since the resistance to mass transfer in the gas phase is much smaller than the resistance in the polymer phase, concentration of each species in the gas phase is assumed to be uniform. In the case of desorption, the transport equation for each cell wall becomes

$$
\frac{\partial C_{p}}{\partial t}=D_{p} \frac{\partial^{2} C_{p}}{\partial x^{2}}
$$

where $C_{p}$ is the concentration of gas in the polymer phase, $D_{p}$ is the diffusivity in the polymer phase and the initial and boundary conditions are

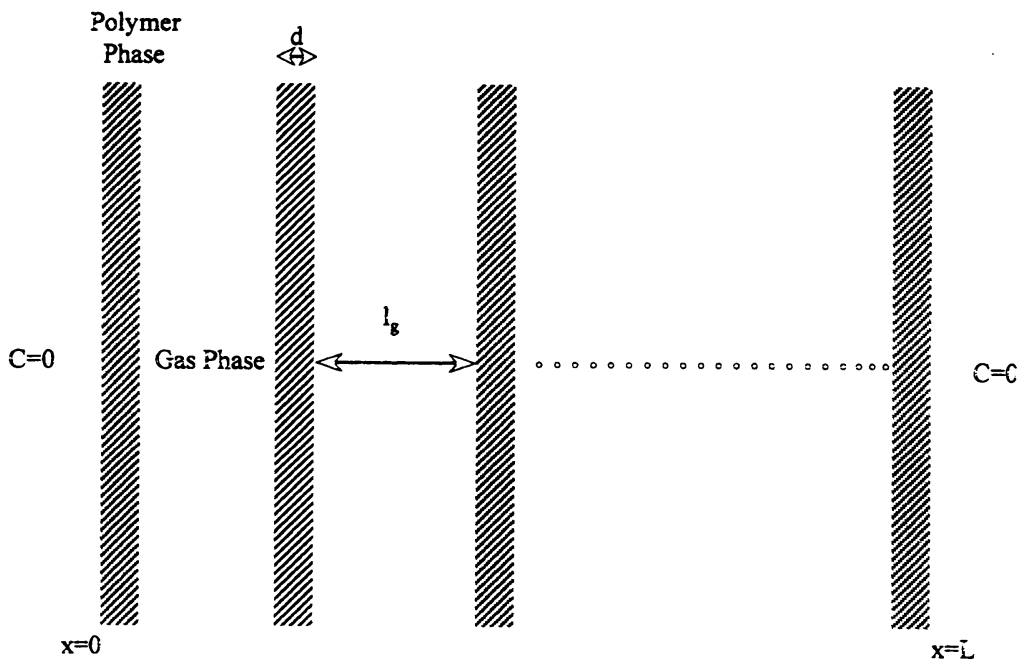

Figure 1. One dimensional cubical foam structure in the unsteady state model. 


$$
\begin{gathered}
C_{p}(x, 0)=C_{p o} \\
C_{p}(0, t)=0 \quad C_{p}(L, t)=0
\end{gathered}
$$

where $C_{p o}$ is the initial concentration of gas in the polymer phase, and $L$ is the thickness of the foam. The transport equation for the gas phase in each cell $i$ can be expressed as follows:

$$
\frac{\partial C_{g}}{\partial t}+\frac{\partial\left(C_{g} v_{g}\right)}{\partial x}=0
$$

where $C_{g}$ is the concentration of gas in the cell $i$, and $v_{g}$ is the velocity of each gas species. Utilizing the assumption that concentration of each species is uniform in the gas phase of each cell, Equation (8) can be integrated from one cell wall to another, and when the integration limits are applied at each polymer gas interface, the following equation is obtained:

$$
A l_{g} \frac{\partial C_{g}}{\partial t}=D_{p} A\left[-\left.\frac{\partial C_{p}}{\partial x}\right|_{i-1}+\left.\frac{\partial C_{p}}{\partial x}\right|_{i+1}\right]
$$

where $A$ is the cross sectional area of the foam and $l_{g}$ is the thickness of the gas phase. An equilibrium relationship exists between the concentration of blowing agent in the polymer and the gas phase at the interface, and is given by the following equation:

$$
C_{g}=K C_{p}
$$

in which $K$ is the partition coefficient. Equation (9) shows that the concentration of gas, $C_{g}$, in the voids changes with time as gas is diffusing through the polymer walls into the cell and is diffusing out of the cell. Initially, the concentration of gas in each cell is $K C_{p o}$. Equations (5) through (10) can be simplified by introducing the following dimensionless quantities:

$$
\begin{array}{rr}
t^{*}=\frac{D_{p} t}{d^{2}} & x^{*}=\frac{x}{d} \\
C^{*}=\frac{C_{p}}{C_{p o}} & C_{g}^{*}=\frac{C_{g}}{K C_{p o}}
\end{array}
$$


where $d$ is the thickness of the cell wall. Combining Equations (10) through (12) with Equations (5) and (9) yields the following dimensionless equations for the polymer and gas phases, respectively.

$$
\begin{gathered}
\frac{\partial C^{*}}{\partial t^{*}}=\frac{\partial^{2} C^{*}}{\partial x^{* 2}} \\
\frac{\partial C_{g}^{*}}{\partial t^{*}}=\frac{d}{K l_{g}}\left[-\left.\frac{\partial C^{*}}{\partial x^{*}}\right|_{i-1}+\left.\frac{\partial C^{*}}{\partial x^{*}}\right|_{i+1}\right]
\end{gathered}
$$

In terms of dimensionless quantities, the initial and boundary conditions can be rewritten as follows:

$$
\begin{array}{cl}
C^{*}\left(x^{*}, 0\right)=1 & C_{g}^{*}\left(x^{*}, 0\right)=1 \\
C^{*}\left(0, t^{*}\right)=0 & C^{*}\left(L, t^{*}\right)=0
\end{array}
$$

In the case of absorption, the same transport equations [Equations (5) and (9)] can be written for the polymer and gas phases with the following modified initial and boundary conditions.

$$
\begin{array}{cc}
C_{p}(x, 0)=0 & C_{g}(x, 0)=0 \\
C_{p}(0, t)=C_{p E} & C_{p}(L, t)=C_{p E}
\end{array}
$$

where $C_{p E}$ is the equilibrium surface concentration. If the following dimensionless quantities are introduced,

$$
\begin{array}{cc}
C^{*}=\frac{C_{p E}-C_{p}}{C_{p E}} & C_{g}^{*}=\frac{C_{g E}-C_{g}}{C_{g E}} \\
t^{*}=\frac{t D_{p}}{d^{2}} & x^{*}=\frac{x}{d}
\end{array}
$$

the same final set of equations [Equations (13) through (16)] are obtained. Therefore, the solution for absorption and desorption becomes equivalent. 


\section{NUMERICAL SOLUTION OF THE EQUATIONS}

Equations (13) and (14) are coupled, and their solution can be obtained numerically using a finite difference approximation. By discretizing these equations and dropping the asterisks for convenience, the following equation can be obtained for the first cell wall:

$$
\frac{C_{k}^{j+1}-C_{k}^{j}}{\Delta t}=\frac{C_{k-1}^{j+1}-2 C_{k}^{j+1}+C_{k+1}^{j+1}}{\Delta x^{2}} \quad 1 \leq k \leq M-1
$$

where $k$ denotes the grid point in the discretized domain, $j$ denotes the time step and $M$ is the number of grid points. The fluxes at the polymer gas interface can be written as follows using the second order Taylor series approximation.

$$
\begin{aligned}
& \left.\frac{\partial C}{\partial x}\right|_{i-1}=\frac{C_{M-2}-4 C_{M-1}+3 C_{M}}{2 \Delta x} \\
& \left.\frac{\partial C}{\partial x}\right|_{i+1}=\frac{4 C_{M+1}-C_{M+2}-3 C_{M}}{2 \Delta x}
\end{aligned}
$$

Similar equations can also be written for other alternating layers. Since surface concentrations are constant and equal, there is a symmetry around the center of the foam, and, therefore, the number of equations to be solved is reduced by a factor of two. For odd number layers (LM/2-1) and for even number layers (LM/2), equations should be solved at each time step. Finally, when all the unknowns are ordered, a linear system of equations, $A x=b$, is obtained where $A$ is the coefficient matrix, $x$ is the unknown vector and $b$ is the right hand side vector. The matrix $A$ is an extremely large sparse matrix with nonzero elements only along a few diagonal lines adjacent to the main diagonal. If the general methods for solving linear systems of equations are used, $N E^{3}$ arithmetic operations are performed each time where $N E$ is the total number of unknowns. It is also wasteful to reserve storage for many zero elements. Considering the fact that $N E$ is very large for the foam structure modeled here, it is impossible to solve the equations in a reasonable computer time, and memory space can also be a 
major problem. However, both computational time and memory can be significantly saved by converting the sparse matrix $A$ to a tridiagonal matrix. The tridiagonal matrix is constructed by defining the fluxes at polymer gas interface as first order approximation in Taylor series. Then, discretized form of the Equation (14) can take the following form:

$$
\frac{C_{M}^{j+1}-C_{M}^{j}}{\Delta t}=\frac{d}{K l_{g}}\left[-\frac{\left(C_{M}^{j+1}-C_{M-1}^{j+1}\right)}{\Delta x}+\frac{\left(C_{M+1}^{j+1}-C_{M}^{j+1}\right)}{\Delta x}\right]
$$

The new tridiagonal coefficient matrix $A$, the unknown vector $x$, and the right hand side vector $b$ are defined as follows:

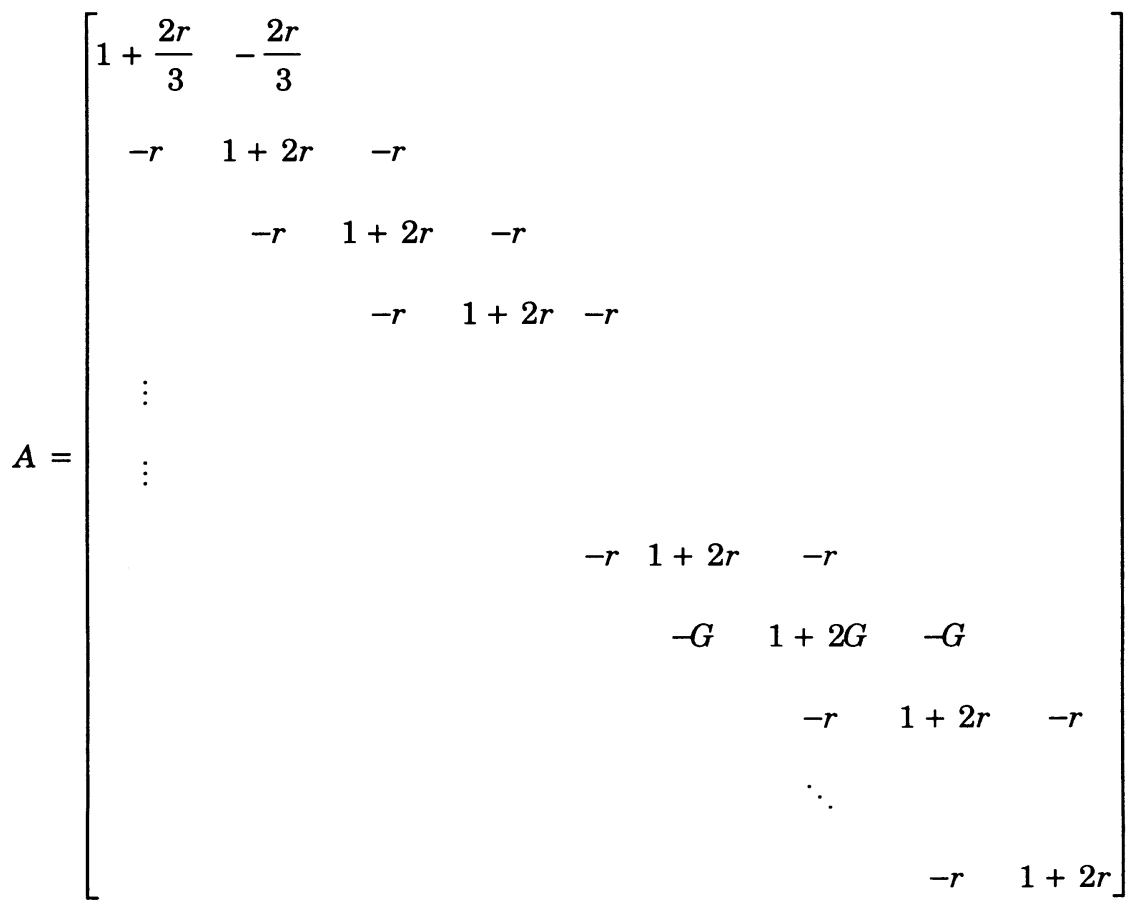

where $r=\Delta t / \Delta x^{2}$ and $G=d \Delta t / K l_{g} \Delta x$ 


$$
x=\left[\begin{array}{c}
C_{1} \\
C_{2} \\
C_{3} \\
C_{4} \\
\vdots \\
\vdots \\
\vdots \\
C_{N}
\end{array}\right] \quad b=\left[\begin{array}{c}
C_{1}^{j} \\
C_{2}^{j} \\
C_{3}^{j} \\
C_{4}^{j} \\
\vdots \\
\vdots \\
\vdots \\
C_{N}^{j}
\end{array}\right]
$$

By converting the sparse matrix to a tridiagonal form, the number of operations are reduced from $N E^{3}$ to $N E$, and the storage space is reduced from $N E^{2}$ to $N E$. Consequently, the large number of equations resulting from the discretized form of the transport equations, written for each polymer and gas phase, can be solved in a reasonable computer time without exploiting available computer memory. The numerical computation was performed on a UNIX-based workstation. The accuracy of the solution was improved by changing $\Delta x$ and $\Delta t$ until no further changes were obtained in the solution vector.

\section{SIMULATION RESULTS}

The predictions from unsteady state model and Bart and Du Cauze De Nazelle model were compared with one set of experimental data available for the PS/ $\mathrm{N}_{2}$ system measured by Booth and Holstein (1993). The comparison is shown in Figure 2. The data was obtained from a gravimetric sorption technique, and it was reported in the paper that the foam had no open cell content before the surface was cut. The open cell content resulting from sample preparation was taken into account by determining the thickness of destroyed surface layer. Authors have confirmed the reliability of their data by evaluating the sample uniformity and the effect of sample thickness on the diffusion process. Predictions from both models compare well with the experimental uptake. It is expected that as the number of cell walls is increased, the rate of aging is slowed down, since cell windows represent most of the barrier to diffusion. To determine the minimum number of cells required to minimize the rate of aging for this foam, transient uptake curves were generated for different numbers of cells. The results are shown in Figure 3. The uptake curves become closer to each other as the number of cells $(N)$ increases, and they will ultimately approach a single curve with a limiting value of $N$. The results indicate that for a PS foam having a thickness of $2.54 \mathrm{~cm}$, at least 2500 cells are required to have the solution which is independent of the number of cells. The foam structure corresponding to $N>$ 


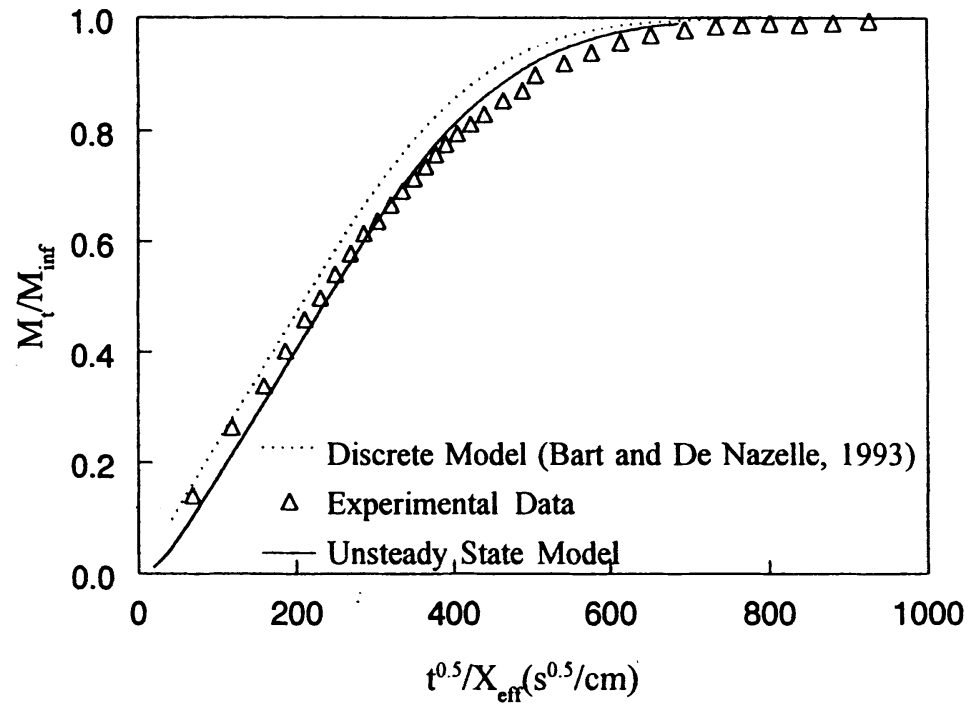

Figure 2. Comparison of the uptake curves for $\mathrm{PS} / \mathrm{N}_{2}$ system. Foam thickness $=2.54 \mathrm{~cm}$.

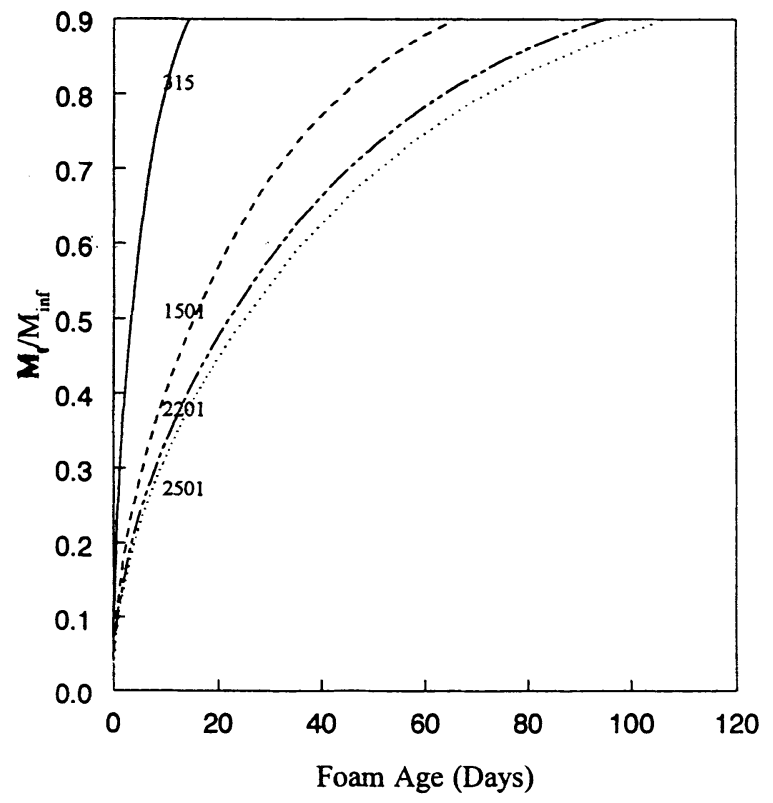

Figure 3. Uptake curves of $\mathrm{PS} / \mathrm{N}_{2}$ system for different number of cells. Foam thickness $=$ $2.54 \mathrm{~cm}$. 
2500 can be treated as a homogeneous medium, and the analytical solution of the continuum model discussed in the first section can be employed to calculate the effective diffusivities. With this in mind, uptake curves were analyzed with the initial slope method (Crank, 1975). From the plot shown in Figure 4, the effective diffusivity of the foam was determined as $5 \times 10^{-7}$ $\mathrm{cm}^{2} / \mathrm{s}$. To evaluate how diffusion is affected by different length scales, uptake curves were determined for a PS foam sample with a thickness of 0.257 $\mathrm{cm}$. Figure 5 shows that the curves are close to each other when the number of cells is changed from 300 to 400 . The effective diffusivity of this sample was determined as $5.1 \times 10^{-7} \mathrm{~cm}^{2} / \mathrm{s}$ from the plot shown in Figure 6 . Thus, the effective diffusivities varied from $5 \times 10^{-7}$ to $5.1 \times 10^{-7} \mathrm{~cm}^{2} / \mathrm{s}$ as the thickness of the sample was reduced by one order of magnitude.

The model can also be used to predict the long term aging behavior and dimensional stability of foams. The factors which influence both aging and dimensional stability can be grouped into four categories as, foam geometry and morphology, foam density, initial cell gas composition and pressure and environmental factors. The morphology of a foam is a complex, position dependent parameter which can be characterized in terms of the following parameters:

- overall density and density distribution

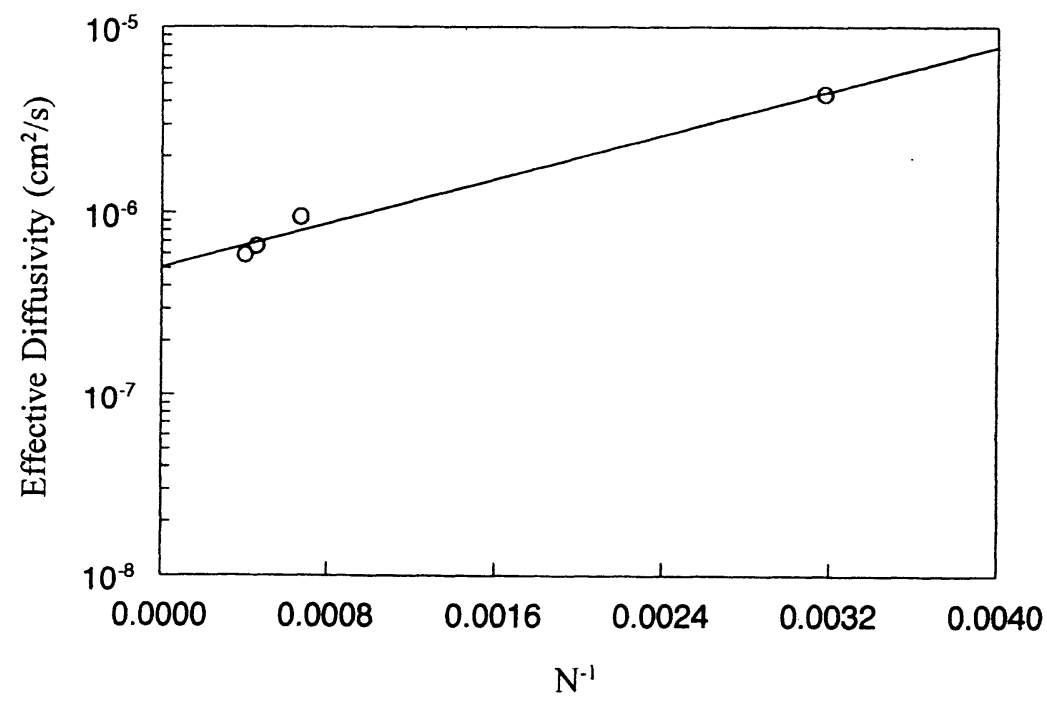

Figure 4. Effective diffusivity of $\mathrm{PS} / \mathrm{N}_{2}$ foam as a function of number of cells. Foam thickness $=2.54 \mathrm{~cm}$. 


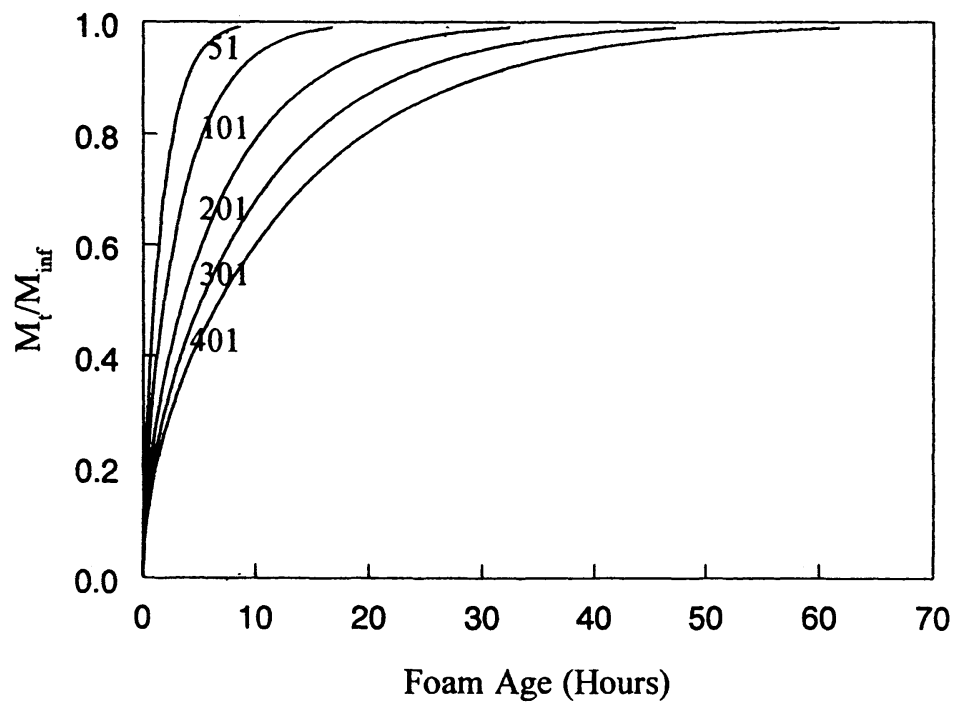

Figure 5. Uptake curves of $\mathrm{PS} / \mathrm{N}_{2}$ system for different number of cells. Foam thickness = $0.257 \mathrm{~cm}$.

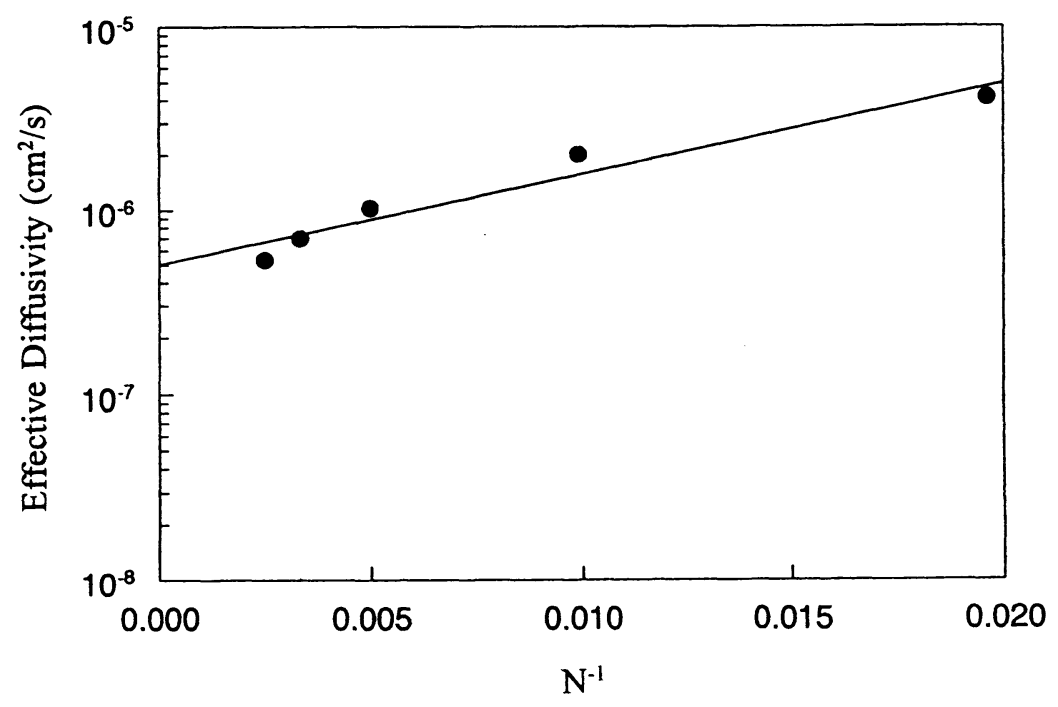

Figure 6. Effective diffusivity of $\mathrm{PS} / \mathrm{N}_{2}$ foam as a function of number of cells. Foam thickness $=0.257 \mathrm{~cm}$. 
- average cell size and cell size distribution

- cell elongation and orientation

- material distribution within the individual cells between struts and windows

Considering that only 2-3 volume percent of a typical foam is polymer, any variation in the way that this material is distributed can have a large impact on the diffusion rates of the different components. To illustrate this phenomena, the effect of changes in the cell size of a PS foam on the rate of aging has been investigated. Figure 7 clearly shows that the rate of aging required to reduce the fraction of blowing agent to $10 \%$ of its initial amount is slowed down dramatically from 2 weeks to 3.5 months as the ratio $l / d$ is decreased from 40 to 4 . From scanning electron microscope pictures of a PU foam, the width of the distribution of cell sizes has been found to decrease with decreasing average cell size (Smiths and Thoen, 1993). This results in a more uniform distribution of material, and consequently in an improvement in the aging. The rate of aging also depends on the initial cell gas composition and pressure.

As an example, the total cell pressure for PU foams blown with different blowing agents was calculated. Figure 8 shows the average partial pressure of CFC-11 and $\mathrm{CO}_{2}$ in a $50 \%$ CFC-11/50\% $\mathrm{CO}_{2}$ blown PU foam. $\mathrm{CO}_{2}$ dif-

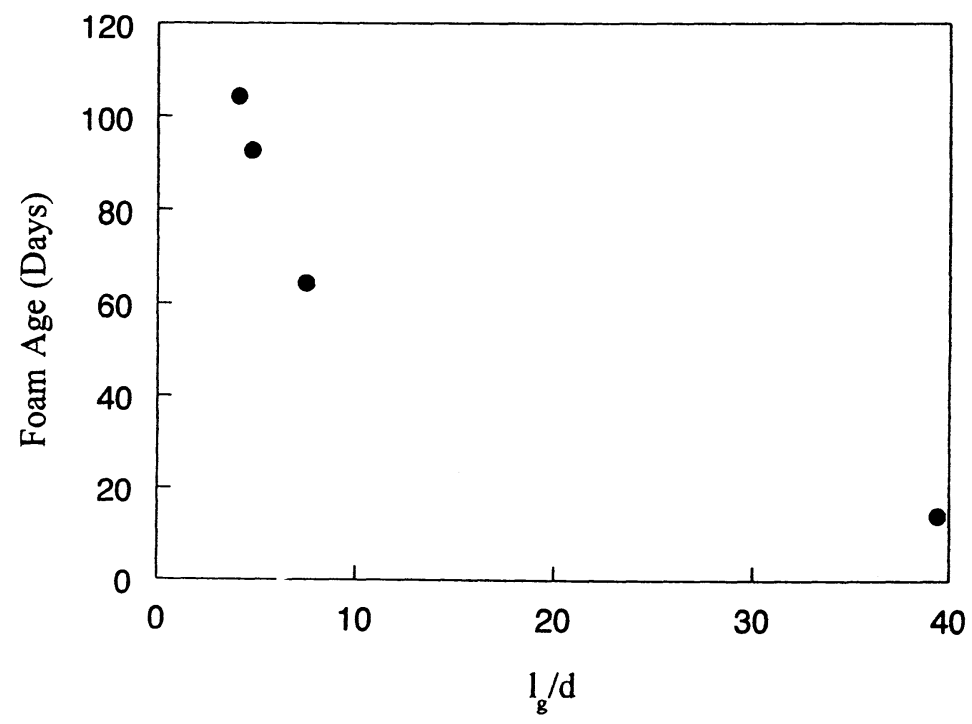

Figure 7. Effect of cell size on foaming aging rate for $\mathrm{PS} / \mathrm{N}_{2}$ system. 


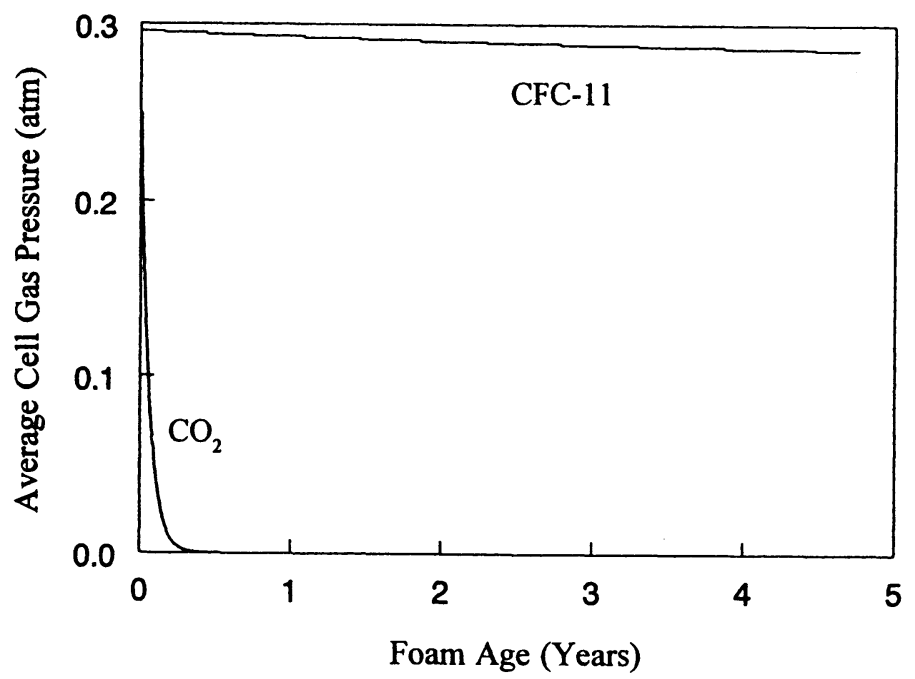

Figure 8. Average cell gas pressure for $50 \% \mathrm{CFC}-11 / 50 \% \mathrm{CO}_{2}$ blown $\mathrm{PU}$ foam.

fuses out of the cells very quickly, and in 190 days all of the $\mathrm{CO}_{2}$ has desorbed. However, only $3.3 \%$ of the CFC-11 is diffused out of the cells in 4.5 years. At the same time, $\mathrm{N}_{2}$ and $\mathrm{O}_{2}$ in air diffuse into the cells. The partial pressures of these components in the cells are shown in Figure 9.

Figure 10 shows the effect of initial cell gas composition on the long term aging behavior of PU foam. For the case of 100\% CFC-11 blown foam, the total pressure increases with time since the diffusion of $\mathrm{N}_{2}$ and $\mathrm{O}_{2}$ into the cells is much faster than the diffusion of CFC-11 out of the cells. This compound has the highest total cell pressure $(\sim 1.4 \mathrm{~atm})$ after 2.5 years of aging as compared to the other two foams. When 50 mole percent of CFC-11 is replaced by carbon dioxide, the cell pressure initially decreases with time, since carbon dioxide diffuses out of the foam faster than the $\mathrm{N}_{2}$ and $\mathrm{O}_{2}$ diffuse in. An initial decrease in the thermal conductivity of the foam is expected due to the rapid loss of $\mathrm{CO}_{2}$, with consequent enrichment of the cell gas with CFC-11. However, overall foam thermal conductivity becomes higher than for the case of $100 \%$ CFC-11 blown foam, since $\mathrm{CO}_{2}$ has a higher thermal conductivity than CFC-11.

In addition to the acceptable aging profile, the dimensional stability of the foam also plays an important role in the optimization process. Foam dimensional stability is essentially the result of a balance between external and internal forces. The external forces are the ambient pressure and the applied load while the internal forces consists of the collapse strength of the foam, the cell pressure, and an increment to the cell pressure as a 


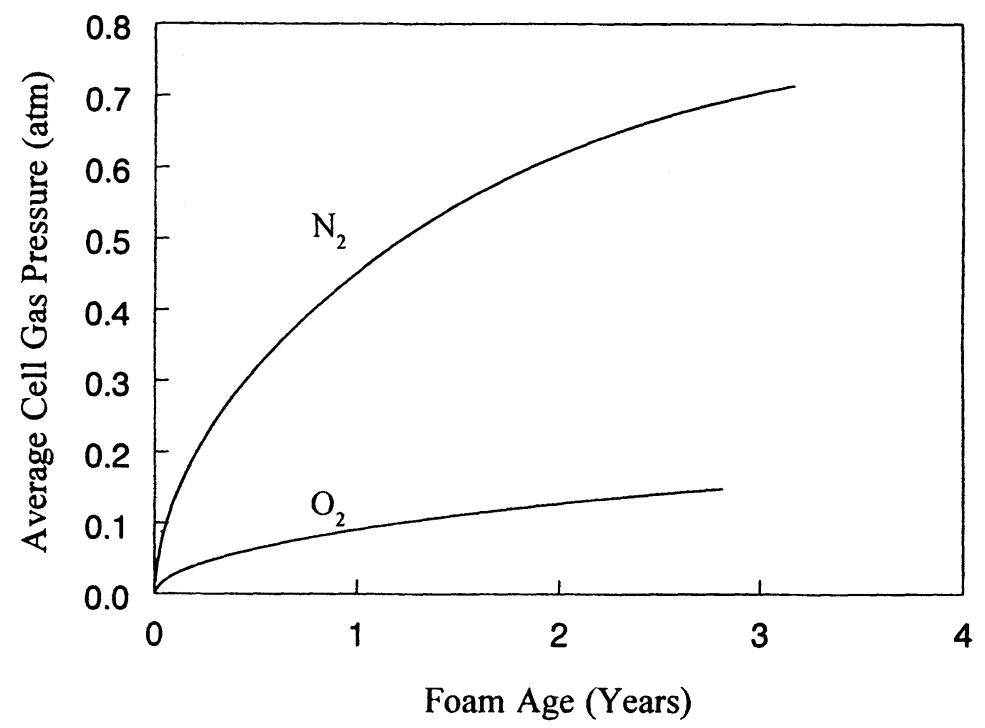

Figure 9. Average cell gas pressure for $50 \%$ CFC-11/50\% $\mathrm{CO}_{2}$ blown $\mathrm{PU}$ foam.

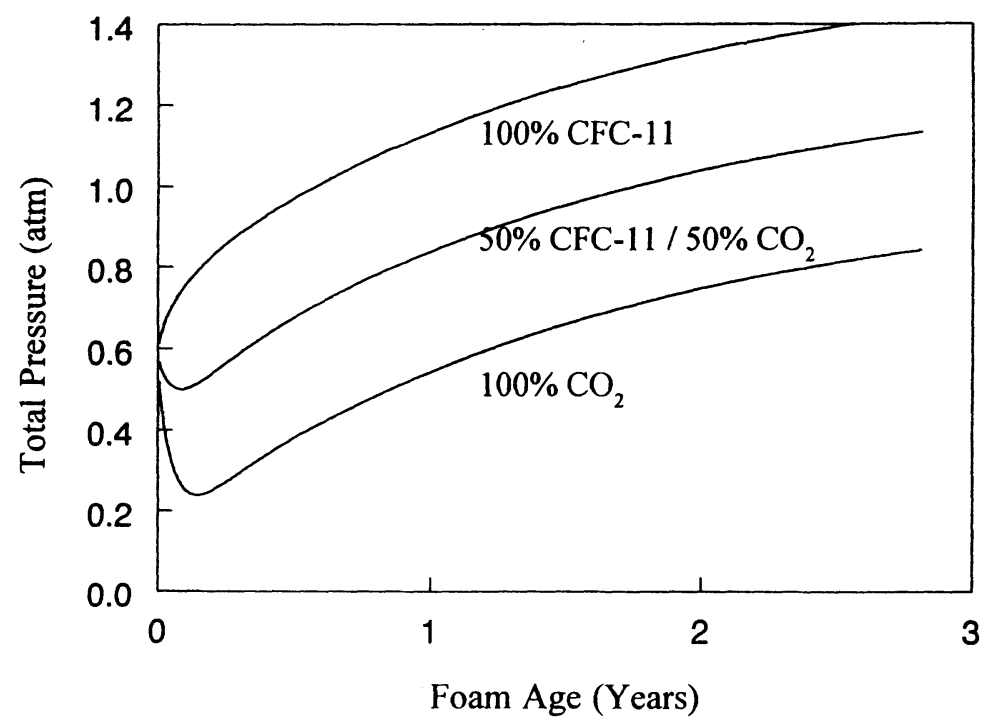

Figure 10. Effect of initial composition on total cell gas pressure for PU foam. 
result of the compression of the sample. Clearly, if the sum of the internal forces exceeds that of the external forces, then the foam will expand; otherwise it will shrink. The actual driving force for any changes in the foam volume is either an increase or decrease in the total cell pressure. As shown in Figure 10, the 100\% CFC-11 blown foam is expected to expand. However, both the 50\% CFC-11 50\% $\mathrm{CO}_{2}$ and $100 \% \mathrm{CO}_{2}$ blown foams show a minima in total cell pressure. These minima have been shown to coincide with the minimum cell strength (Daems et al., 1994), i.e., it is a measure of the dimensional stability of the foam. The PU foam blown with $50 \%$ CFC-11/50\% $\mathrm{CO}_{2}$ shows some potential for shrinkage within a month after its production. In the case of a fully $\mathrm{CO}_{2}$ blown foam, a severe dimensional stability problem is expected because of the significant minimum in cell pressure after 2 months.

The strength of the foam is also affected by the plasticization of the polymer matrix as a result of the distribution of the blowing agent or soluble additives between the polymer and gas phases. Concentrations of CFC-11 and $\mathrm{CO}_{2}$ in the gas and polymer phases are shown in Figures 11 and 12 respectively. Most of the CFC-11 in the foam is dissolved in the polymer phase. A significant amount of $\mathrm{CO}_{2}$ is also dissolved in the polymer phase, but its solubility is not as high as the solubility of CFC-11. $\mathrm{N}_{2}$ and $\mathrm{O}_{2}$ both have negligible solubility in the polymer phase as shown in Figures 13 and 14 re-

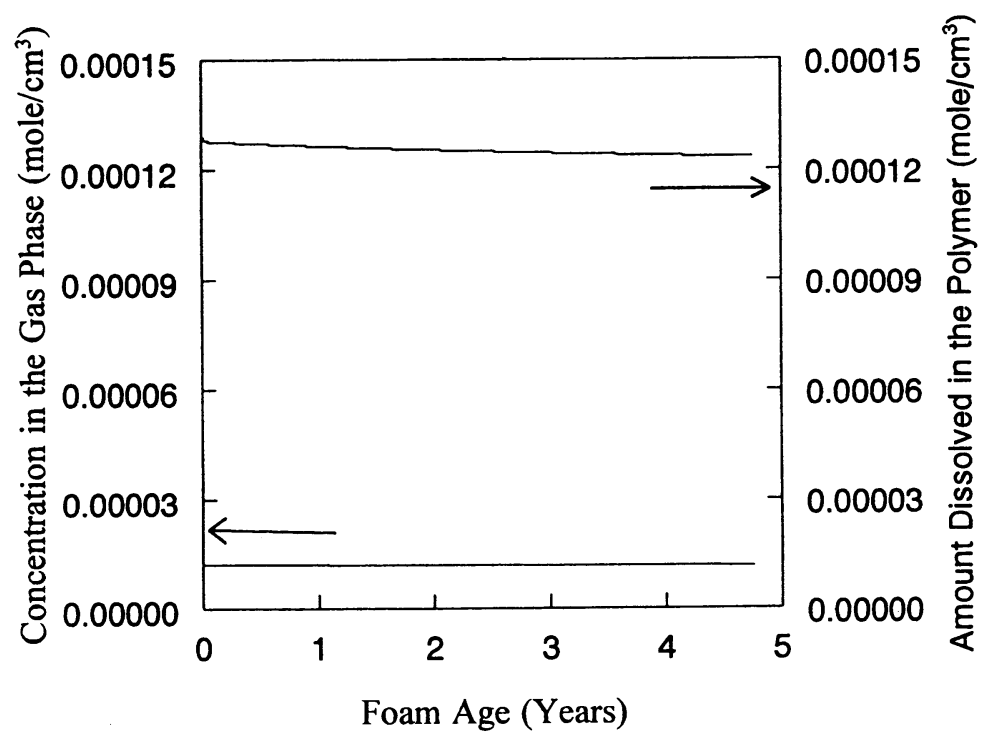

Figure 11. Distribution of CFC-11 between gas and polymer phases in PU foam. 


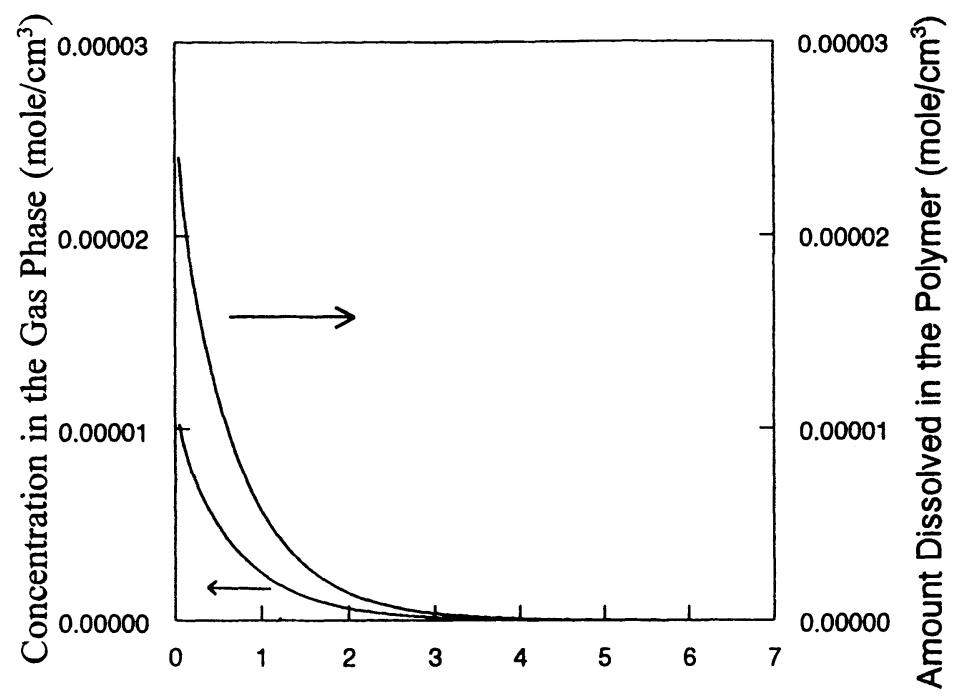

Foam Age (Months)

Figure 12. Distribution of $\mathrm{CO}_{2}$ between gas and polymer phases in $\mathrm{PU}$ foam.

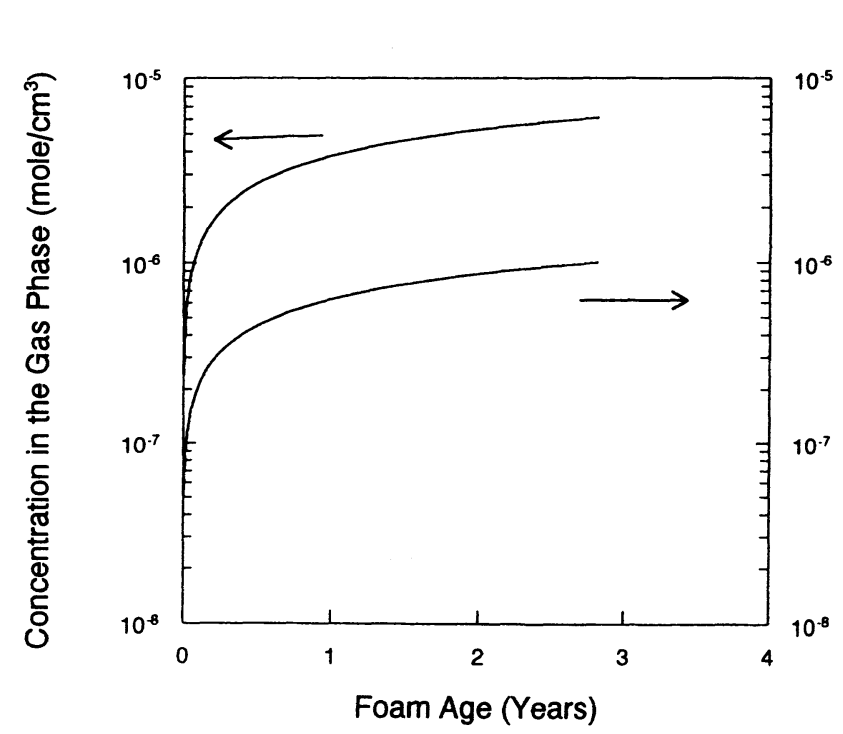

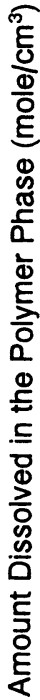

Figure 13. Distribution of $\mathrm{N}_{2}$ between gas and polymer phases in $\mathrm{PU}$ foam. 


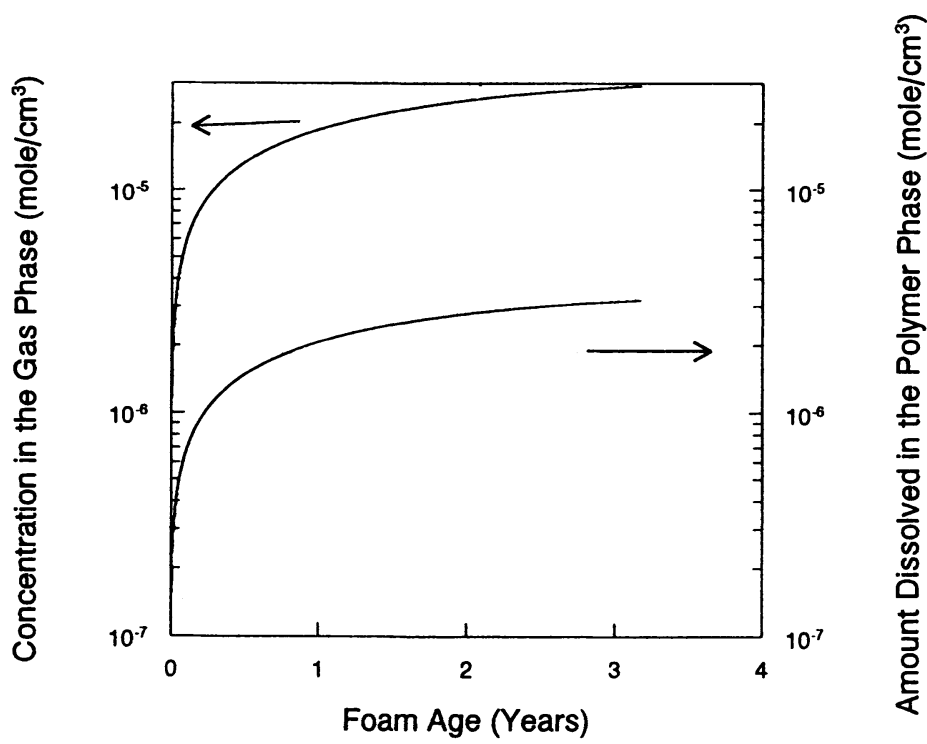

Figure 14. Distribution of $\mathrm{O}_{2}$ between gas and polymer phases in PU foam.

spectively. From these results, it is apparent that one cannot accurately model the effective solubility of the foam as the solubility of gas only in the void space. For improved predictions, the solubility of different components in the polymer matrix should be taken into account.

\section{CONCLUSION}

The models available in the literature to describe diffusion in closed cell foams have been reviewed. The effective diffusivities were predicted from a discrete model developed by Bart and Du Cauze De Nazelle (1993), and the results have shown that these values are not comparable with the experimentally measured diffusivities. The large discrepancy can be partly explained by the presence of struts and open cells in the cell walls. Another major reason for the poor predictability of this model is related to its simplified assumptions. The relationship derived for effective diffusivity of a foam is restricted with steady-state condition and constant diffusivity in the polymer phase and linear equilibrium relationship given by Henry's law. Although simulation results presented in this work are based on linear equilibrium relationships and constant diffusivity in the polymer phase, the unsteady-state model has the potential to incorporate a nonlinear sorption isotherm (Vrentas and Vrentas, 1991) and concen- 
tration dependent diffusion coefficients. This is the major advantage of the unsteady-state model over the discrete model. If these two complications are included in the discrete model, it is not possible to derive an explicit relationship for the effective diffusivity. However, the incorporation of these effects into the unsteady state model again results in a simple tridiagonal matrix, but the coefficients of this matrix are not constant, and hence a solution could involve an iterative technique.

An efficient numerical solution algorithm was developed to solve extremely large number of equations in a reasonable computational time without crashing the available computer memory. Therefore, the unsteady state model can be used to predict the effective diffusivity, long term aging profile, and dimensional stability of foams. It has the added advantage of being able to find the optimum conditions to minimize the rate of aging and maximize the dimensional stability of the foams. For accurate predictions, the thickness of the cell wall or percent of polymer at the cell walls and the average distance between the cell walls should be determined experimentally. It has been observed that the morphological state significantly affects both transport and equilibrium properties. Therefore, in the predictions, diffusivity and solubility data measured along the cell walls should be used instead of the data obtained from the films.

\section{REFERENCES}

Bart, G.C.J., Du Cauze De Nazelle, M.R., 1993, J. Cellular Plastics, vol:29, p:29.

Bhattacharjee, D., Booth, J.R., 1995. J. Cellular Plastics, vol:31, p:244.

Bomberg, M., 1988, J. Cellular Plastics, vol:24, p:327.

Booth, J.R., Holstein, T.J., 1993, 8th International Conference on Thermal Insulation, $\mathrm{p}: 246$.

Booth, J.R., 1993, 3rd International Workshop on Long-Term Thermal Performance of Cellular Plastics.

Brandreth, D.A., Ingersoll, H.G., 1980, J. Cellular Plastics, (July/August) p:235.

Brandreth, D.A., 1989, J. Cellular Plastics, vol:25, p:139.

Brodt, K.H., Brood, R.R.H., Bart, G.C.J., 1995, J. Thermal Insul. and Bldg. Envs., vol:19, p:132.

Crank, J., 1975, The Mathematics of Diffusion, 2nd Edition, Oxford, Clarendon Press, London.

Cuddihy, E.F., Moacanin, J., 1967, J. Cellular Plastics, (February), p:73.

Cuddihy, E.F., 1976, J. Cellular Plastics, (March/April), p:114.

Daems, D., Rosbotham, D., Singh, S.N., Franco, M.V., 1994, 35th Annual Polyurethane Technical/Marketing Conference, p:428.

Duda, J.L., Vrentas, J.S., 1970, Encyclopedia of Polymer Science and Technology, Vol: 13 John Wiley and Sons, New York.

Dwyer, F.J., Zwolinski, L.M., Thrun, K.M., 1990, Plastics Engineering, p:29. 
Fleming, G.K., Koros, W.J., Macromolecules, vol:19, p:2285.

Jarfelt, U., 1994, UTECH'94, Paper 23, p:1.

Koros, W.J., Paul, D.R., Rocha, A.A., 1976, J. Poly. Sci., Phys. Ed., vol:14, p:687.

Koros, W.J., Paul, D.R.J.L., 1978, J. Poly. Sci., Poly. Phys. Ed., vol:16, p:1947.

Lee, W.M., 1973, J. Cellular Plastics, (May/June), p:125.

Norton, F.J., 1967, J. Cellular Plastics, (January), p:23.

Norton, F.J., 1982, J. Cellular Plastics, (September/October), p:300.

Ostrogorsky, A.G., Glicksman, L.R., Reitz, D.W., 1984, American Society of Mechanical Engineers, New Orleans, LA, USA, Paper:84-WA/HT-98, p:1.

Ostrogorsky, A.G., Glicksman, L.R., Reitz, D.W., 1986, J. Heat and Mass Transfer, vol:29, p:1169.

Ostrogorsky, A.G., Glicksman, L.R., 1988a, J. Cellular Plastics, vol:24, p:215.

Ostrogorsky, A.G., Glicksman, L.R., 1988b, Transactions of ASME, vol:110, p:500.

Park, C.P., 1993, Nonmunjip-Sekye Hanminchok Kwahak Kisulcha Jonghap Haksul Taehoe, p:1076.

Reitz, D.W., Scheutz, M.A., Glicksman, L.R., 1984, J. Cellular Plastics, (MarchApril), p:104.

Shankland, I.R., 1990, Insulation Materials, Testing, and Applications, ASTM STP 1030, D.L. McElroy and J.F. Kimpflen, eds., Philadelphia: ASTM, p:174.

Smiths, G.F., Thoen, J.A., 1993, J. Cellular Plastics, vol:29, p:57.

Toi, K., Paul, D.R., 1982, Macromolecules, vol:15, p:1104.

Vieth, W.R., Tam, P.M., Michaels, A.S., 1966, J. Colloid and Interface Sci., vol:22, $\mathrm{p}: 360$.

Vrentas, J.S., Vrentas, C.M., 1991, Macromolecules, vol:24, p:2404.

\section{BIOGRAPHY}

\section{Sacide Alsoy}

Sacide Alsoy received her Bachelor and Master degrees in Chemical Engineering from Ege University, Turkey. She obtained her Ph.D. degree in Chemical Engineering from The Pennsylvania State University. Following a short period of working at Penn State, she joined Izmir Institute of Technology, Turkey, as an assistant professor. 